\title{
Lichen Simplex Chronicus of the Conjunctiva
}

\author{
Heather A Potter, MD' ${ }^{1}$, Vivian Lee, MD , Molly A Hinshaw, MD², Sherif S Khedr, MD ${ }^{1}$, Daniel \\ M Albert, $\mathbf{M D}^{1}$, and Cat $\mathbf{N}$ Burkat, $\mathbf{M D}^{1}$ \\ ${ }^{1}$ University of Wisconsin, Department of Ophthalmology and Visual Sciences \\ ${ }^{2}$ University of Wisconsin, Department of Dermatology
}

\section{Keywords}

Lichen simplex chronicus; psychogenic excoriation; conjunctival metaplasia

\section{To the Editor}

Lichen simplex chronicus (LSC) is a disorder commonly encountered by dermatologists, caused by constant rubbing of the skin that clinically induces thickening and lichenification. While a case report of LSC of the eyelids has been published (1), a careful search of the literature did not disclose any prior case of LSC of the palpebral conjunctiva.

\section{Case Description}

A 58-year-old woman presented to the ophthalmology service at the University of Wisconsin with a chief complaint of left eye foreign body sensation and redness for two months. The patient reported that an outside ophthalmologist had given her a prescription for gentamycin ophthalmic ointment for treatment of bacterial conjunctivitis, but she developed extreme itchiness after using the ointment for a few days and presented for a second opinion.

Her past history includes bipolar disorder and multiple corneal ulcers. Best-corrected visual acuity was 20/60 in the right eye and light perception in the left. Intraocular pressures and pupils were normal. She had diffusely erythematous and thickened left upper and lower eyelids. The left upper eyelid margin was rounded with loss of normal architecture and was severely floppy with imbrication of the upper lid over the lower lid margin. Broken eyelashes were noted. With eversion of her left upper eyelid, a lichenified palpebral conjunctiva was seen (Figure 1). The right eyelids were unremarkable. Marginal reflex distance 1 was $4 \mathrm{~mm}$ on the right and $0 \mathrm{~mm}$ on the left, and levator function was $18 \mathrm{~mm}$ and $14 \mathrm{~mm}$. She also had a scarred, vascularized cornea in the left eye. Concerned for malignancy, an elliptical incisional biopsy of the left palpebral conjunctiva was performed and submitted to pathology. Microscopic examination demonstrated a metaplastic conjunctiva showing significant acanthosis and squamatization with uniform keratinocytes exhibiting compact ortho-and parakeratosis (Figure 2). A mild lymphocytic infiltrate was seen in the substantia propria.

Corresponding Author: Vivian Lee, MD, University of Wisconsin 600 Highland Avenue F4/344 CSC Madison, WI 53792 608-262-4800608-263-0543, vlee@ophth.wisc.edu.

Please send reprints to: Vivian Lee, MD, University of Wisconsin, Department of Ophthalmology and Visual Sciences, c/o Ms.

Michele Kempfer, 600 Highland Avenue, F4/344 CSC, Madison, WI 53792 
Although the patient initially denied touching her eye, she eventually admitted to rubbing the inside of her left upper eyelid with a cotton tip applicator and plucking and shaving her eyelashes because of long-standing itchiness. The diagnoses of LSC, trichotillomania, rubbing-induced lax upper eyelid, and ptosis were made. Topical steroids were prescribed, but the patient was noncompliant. She agreed instead to a pentagonal wedge resection and ectropion repair for the lax eyelid, and external levator repair for ptosis. The pentagonal wedge resection specimen microscopically showed stratified squamous epithelium exhibiting orthokeratosis, parakeratosis, acanthosis, and irregular hyperplasia of the rete pegs. Fibrotic tissue and chronic inflammation were present in the tarsal plate with atrophic meibomian glands and one showing granulomatous inflammation. Verhöeff van Gieson stain demonstrated decreased elastin in the tarsal plate. At 4 weeks postoperatively, the patient developed a small dehiscence of her incision from resumed rubbing; and at 12 weeks, some of the laxity in her eyelid had returned.

We believe this case to be one extreme example of metaplastic transformation of the conjunctival epithelium encountered in our department, exemplifying the chronic changes that can occur from mechanical rubbing of the conjunctiva. The patient's palpebral conjunctiva demonstrated thickening, lichenification, and histologic changes consistent with LSC. Pathologic findings in this entity include orthokeratosis, parakeratosis, acanthosis, irregular elongation of the rete pegs, hypergranulosis, and alterations in the cellular population of the stroma (2). The severity of this case likely stems from a vicious cycle of ocular injury. Incited by itchiness, the patient rubbed her palpebral conjunctiva for relief, thereby inducing lax eyelid syndrome (LES), which exacerbated and perpetuated her initial symptom of itchiness. While LES also exhibits similar histologic findings to LSC, including papillary hyperplasia, keratinization, and epidermalization of the tarsal conjunctiva (3-4), LSC is likely the predominant cause of the patient's findings since she denied sleeping on her left side and the severity of the metaplastic transformation. Although horizontal shortening surgical procedures have been shown to resolve the symptoms of lax eyelid syndrome and reverse clinical findings (3), patients often have a history of psychological disorders in cases of this severity, particularly mood and anxiety disorders, as in our patient (5). Our patient also suffered from trichotillomania, characterized by repetitive hair pulling that results in noticeable hair loss in absence of other signs of disease (6).

While disorders such as LSC are not commonly encountered by ophthalmologists, the few cases that do occur can be a diagnostic puzzle, particularly since patients usually deny any self-inflicted injuries or behaviors. A detailed history is required that often includes interviews from family members (5). Treatment is complex and usually requires counseling, mood stabilizers, and surgery. When involving the lids or conjunctiva, a multi-disciplinary approach is required, including both ophthalmology and psychiatry (5).

\section{Acknowledgments}

Financial Support: None

We are grateful for the support from the McPherson Eye Research Institute at the University of Wisconsin and Core Grant for Vision Research P30EY016665

\section{Cited References}

1. Ferry AP, Kaltreider SA. Lichen simplex chronicus of the eyelid. Arch Ophthalmol. 1999; 117(6): 829-831. [PubMed: 10369600]

2. Bolognia, JL.; Jorizzo, JL.; Rapini, RP. Dermatology. Mosby; 2003.

3. Burkat CN, Lemke BN. Acquired lax eyelid syndrome: an unrecognized cause of the chronically irritated eye. Ophthal Plast Reconstr Surg. 2005; 21(1):52-58. 
4. van den Bosch WA, Lemij HG. The lax eyelid syndrome. Br J Ophthalmol. 1994; 78(9):666-670. [PubMed: 7947544]

5. Arnold LM, Auchenbach MB, McElroy SL. Psychogenic excoriation. Clinical features, proposed diagnostic criteria, epidemiology and approaches to treatment. CNS Drugs. 2001; 15(5):351-359. [PubMed: 11475941]

6. Mawn LA, Jordan DR. Trichotillomania. Ophthalmology. 1997; 104(12):2175-2178. [PubMed: 9400781] 


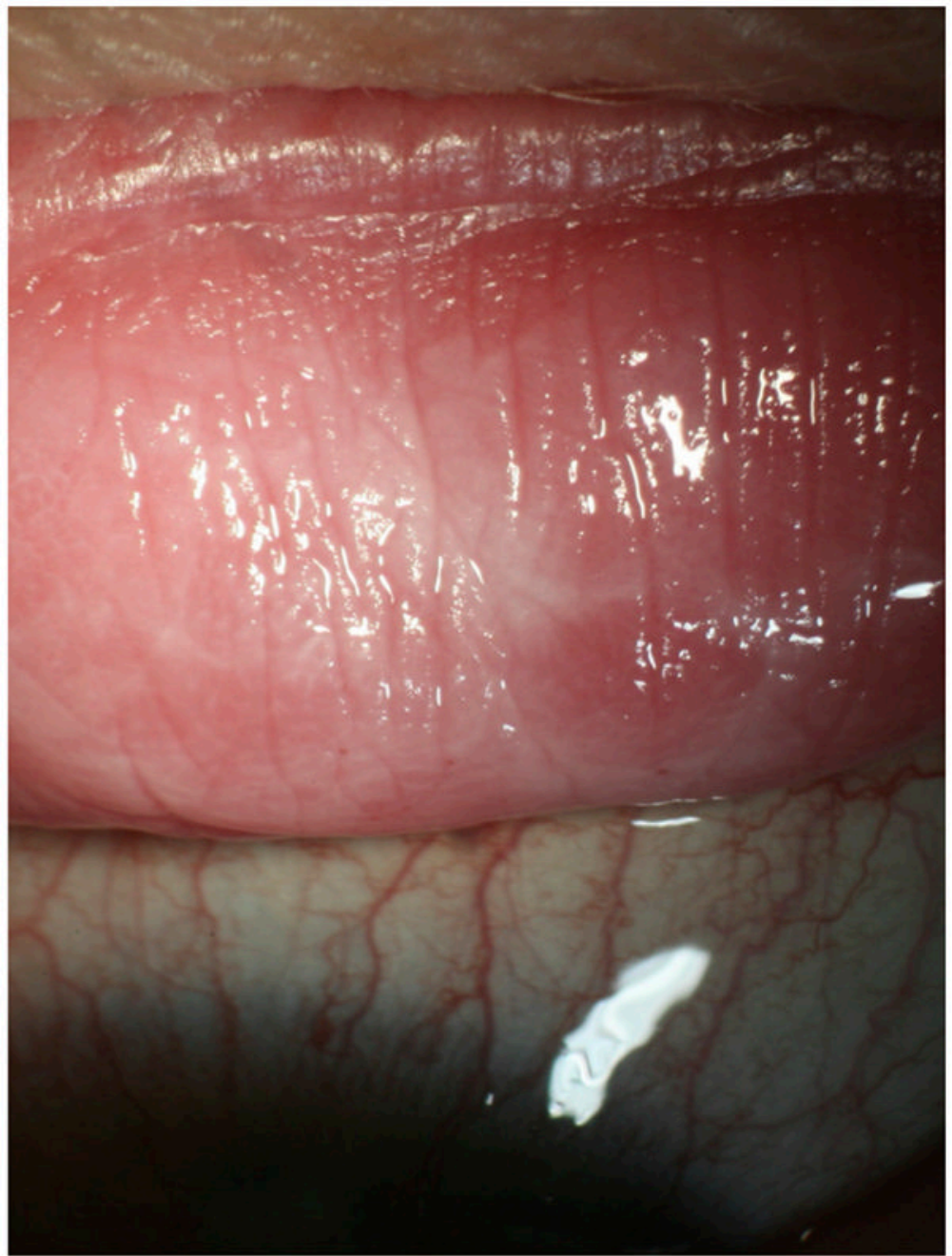

Figure 1.

Slit lamp photograph of patient's everted left upper eyelid, revealing a thickened and keratinized palpebral conjunctiva. [Photo courtesy of Cat N. Burkat, MD] 


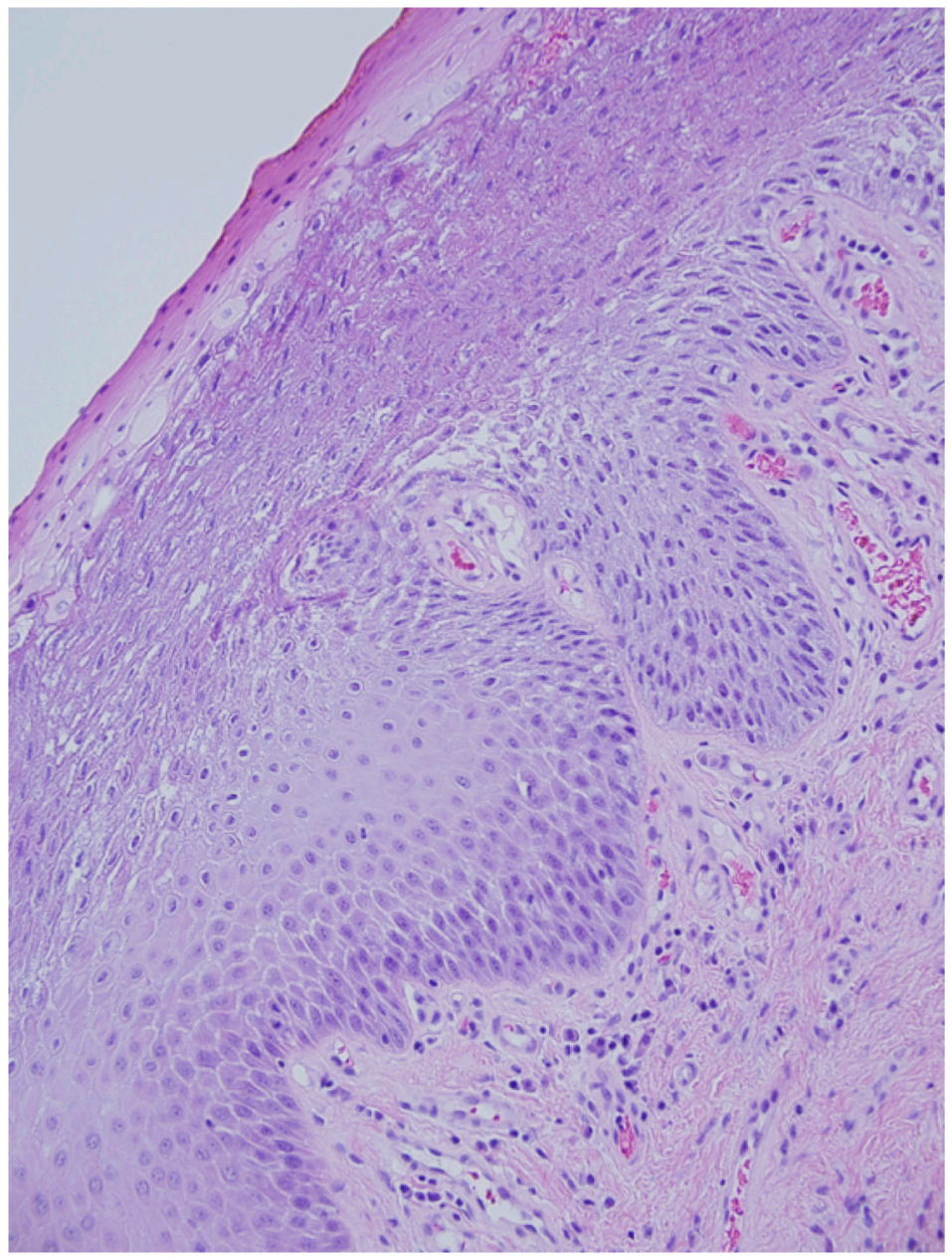

Figure 2.

Elliptical incisional biopsy of the left palpebral conjunctiva demonstrates microscopically a metaplastic conjunctiva showing significant acanthosis, ortho- and parakeratosis with squamatization. Chronic non-granulomatous inflammation is present in the substantia propria. (H\&E x 200) [Photo courtesy of Vivian Lee, MD] 